\title{
Prognostic value of predischarge 12 lead electrocardiogram after myocardial infarction compared with other routine clinical variables
}

\author{
PAOLO FIORETTI, JAN G P TIJSSEN, AIDA J AZAR, ETTORE LAZZERONI, * \\ R W BROWER, $\dagger$ HARALD J TEN KATEN, $\dagger$ JACOBUS LUBSEN, \\ PAUL G HUGENHOLTZ
}

From the Thoraxcenter, Erasmus University, Rotterdam, the †Interuniversity Cardiology Institute, Rotterdam, the Netherlands, and ${ }^{\star}$ Divisione di Cardiologia, Ospedale Regionale, Parma, Italy

SUMMARY The prognostic value of QRS score (Selvester), ST depression, ST elevation, extra- 윽 systoles, $P$ terminal force in V1, and QTc derived from the predischarge 12 lead electro- $\rightarrow$ cardiogram was assessed after myocardial infarction in 474 patients without intraventricular conduction defects, ventricular hypertrophy, or atrial fibrillation. The usefulness of these results $\vec{\bullet}$ in risk assessment was compared with that of other clinical data. During follow up 45 patients died. Logistic regression analysis showed that QRS score, ST depression, and QTc were independently predictive of cardiac mortality. When multivariate analysis was applied to clinical and electrocardiographic data together, however, the 12 lead electrocardiogram did not provide independent information additional to that provided by other routine clinical findings and laboratory tests such as a history of previous myocardial infarction, clinical signs of persistent heart failure, indication for digitalis or antiarrhythmic drugs at discharge, and enlarged heart on chest $x$ ray.

In conclusion, the electrocardiogram has important prognostic value; however, it is not powerful enough to further improve the risk assessment of post-infarction patients.

Some tests of cardiac function (mainly related to the detection of myocardial ischaemia, the quality of left ventricular function, or the occurrence of ventricular arrhythmias) have been used to assess risk after myocardial infarction. It is apparent that there is considerable overlap in the information derived from these tests. ${ }^{1}$ Furthermore, because many hospitals cannot provide all these tests, which are expensive and in some cases risky for the patient, it is important to know how to make the best possible use of standard clinical and laboratory tests in risk assessments.

The 12 lead electrocardiogram is valuable in risk assessment in patients after myocardial in-

Requests for reprints to Dr Paolo Fioretti, Erasmus University, Thoraxcenter BD 373, PO Box 1738, 3000 DR Rotterdam, The Netherlands.

Accepted for publication 24 November 1986 farction. ${ }^{2-7}$ However, few studies have also determined the usefulness of other clinical findings ${ }^{2}$ or have used a multivariate analysis for variables that predict mortality. The routine 12 lead electrocardiogram is an attractive tool for risk assessment $O$ in patients after infarction because it provides information on the extent of myocardial damage, 8 음 left atrium overload, ${ }^{79}$ presence of myocardial ischaemia, ${ }^{210}$ arrhythmias, ${ }^{3}$ and electrical $\%$ instability. 45

The aim of this study was to analyse the power of N different variables extracted from the predischarge $\sigma$ electrocardiogram to predict one year survival after myocardial infarction and to establish whether they provide information that is additional to that sup- $\stackrel{5}{-}$ plied by routine clinical findings and tests. The 0 study was based on 474 hospital survivors of myocardial infarction without intraventricular conduc- $\mathbb{D}$ tion defects, ventricular hypertrophy, or atrial这 fibrillation-all of which are markers of increased cardiac risk. 
Table 1 Data on the patients in whom clinical and electrocardiographic variables were studied

\begin{tabular}{|c|c|c|}
\hline & No & 1 year mortality \\
\hline Admitted with AMI & 706 & $174(25 \%)$ \\
\hline Hospital deaths & 104 & $104(100 \%)$ \\
\hline Discharged alive & 602 & $72(12 \%)$ \\
\hline Excluded: & 128 & $27(21 \%)$ \\
\hline Lost to follow up & 6 & - \\
\hline Non-cardiac death & 2 & 2 \\
\hline Missing or bad quality & & \\
\hline predischarge ECG & 36 & $3(8 \%)$ \\
\hline IVCD and/or VH and/or AF & 84 & $22(26 \%)$ \\
\hline Study base & 474 & $45(9 \%)$ \\
\hline
\end{tabular}

AMI, acute myocardial infarction; ECG, 12 lead electrocardiogram; IVCD, intraventricular conduction defects; VH, ventricular hypertrophy; $\mathrm{AF}$, atrial fibrillation.

\section{Patients and methods}

From March 1981 to December 1983, 706 patients were consecutively admitted to the coronary care unit of the Thoraxcenter with a confirmed diagnosis of acute myocardial infarction. Four hundred and seventy four of the 602 hospital survivors form the base for this study (table 1). All these patients were followed for one year, with death as the end point. Table 2 shows the differences in selected baseline characteristics between 474 patients included in the study and 84 patients excluded because they had intraventricular conduction defects, ventricular hypertrophy, or atrial fibrillation. We used two sets of information to predict mortality-one derived from the predischarge 12 lead electrocardiogram and another based on the remaining clinical and laboratory findings obtained in hospital.

\section{ELECTROCARDIOGRAPHIC VARIABLES}

At discharge the standard 12 lead electrocardiogram was obtained on a three channel Hewlett Packard $1513 \mathrm{~A}$ recorder at a paper speed of $25 \mathrm{~mm} / \mathrm{s}$. The following electrocardiographic variables were measured: the presence of ventricular extrasystoles, QRS score developed by Selvester, ${ }^{8}$ ST depression (horizontal or downsloping) or elevation of $\geqslant 1 \mathrm{~mm}$, $\mathrm{P}$ terminal force in $\mathrm{V} 1,{ }^{79}$ and QT interval corrected for heart rate $(\mathrm{QTC}){ }^{45}$ The measurements were performed by one cardiologist who was unaware of the outcome of the individual patients. In a previous study we showed that when the QRS score was measured agreement between two independent observers was good. ${ }^{6}$

\section{OTHER CLINICAL VARIABLES}

The other routine variables were age, sex, history of previous myocardial infarction, history of previous stable angina pectoris, peak serum creatine kinase, the worst Killip class while the patient was in the coronary care unit, early post-infarction angina, "late" ( $\geqslant 72$ hours after the onset of infarction) ventricular fibrillation, late sustained ventricular tachycardia or atrial arrhythmias, persistent heart failure after discharge from the coronary care unit, resting heart rate at discharge, cardiomegaly (cardiothoracic ratio $>50 \%$ ), and signs of pulmonary congestion on predischarge chest $x$ ray, and medication at discharge (digitalis, diuretics, $\beta$ blockers, antiarrhythmics, anticoagulants). Selection of these variables was based on clinical experience and previous research in our clinic.

Other tests performed before discharge such as radionuclide ventriculography at rest (in 417 patients), symptom limited bicycle ergometry (in 340 patients), and 24 hour ambulatory electrocardiographic monitoring (in 310 patients), have been described elsewhere. ${ }^{1}$ Cardiac catheterisation with coronary arteriography was performed in 235 patients as an elective procedure or as part of a randomised trial of intracoronary streptokinase. ${ }^{11-13}$ Before discharge from hospital 46 patients had coronary artery bypass surgery, and 15 had percutaneous transluminal coronary angioplasty.

\section{STATISTICAL ANALYSIS}

Univariate analysis of the different electrocardiographic and clinical variables was performed. Continuous variables were divided into thirds. The 95\% confidence intervals for the respective risk ratios were calculated according to Miettinen and Nurminen ${ }^{10}$; the first tertile was regarded as the reference group. If the $95 \%$ confidence interval exceeds 1 , the association of the respective variable with the risk of dying within one year is statistically significant at the 5\% level.

In the multivariate analysis the logistic regression model was used to predict mortality. Thus the objective was to find the combination of variables that most closely predicted mortality. As a general principle, we used indicator variables. These variables

Table 2 Differences of selected baseline characteristics between patients in the study group and those excluded from the study because of ventricular conduction defects, atrial fibrillation, or ventricular hypertrophy

\begin{tabular}{|c|c|c|c|}
\hline & Study group & Excluded & pvalue \\
\hline $\begin{array}{l}\text { No of patients } \\
\text { Age (yr) mean (SD) } \\
\text { Previous AMI (No }(\%)) \\
\text { Late heart failure }(\text { No }(\%)) \\
\text { CTR }>50 \%(\text { No }(\%)) \\
\text { EF }(\%(\text { mean }(\text { SD }))) \\
\text { Extrasystoles }(\text { No }(\%)) \\
\text { PTF (mm ms (mean (SD))) }\end{array}$ & $\begin{array}{l}474 \\
57(11) \\
143(30) \\
63(13) \\
95(20) \\
47(14) \\
19(4) \\
20(4)\end{array}$ & $\begin{array}{l}84 \\
61(10) \\
26(33) \\
27(35) \\
34(44) \\
37(17) \\
6(8) \\
26(18)\end{array}$ & $\begin{array}{l}\overline{0} \\
\text { N.002 } \\
\text { NS } \\
0.0005 \\
0.0005 \\
0.0005 \\
\text { NS } \\
0.0005\end{array}$ \\
\hline
\end{tabular}

AMI, acute myocardial infarction; CTR, cardiothoracic ratio; EF, left ventricular ejection fraction; PTF, terminal force of $P$ wave in $\mathrm{V} 1$. 
Table 3 Mortality, risk ratios, and confidence intervals for the different electrocardiographic and clinical variables determined by univariate analysis

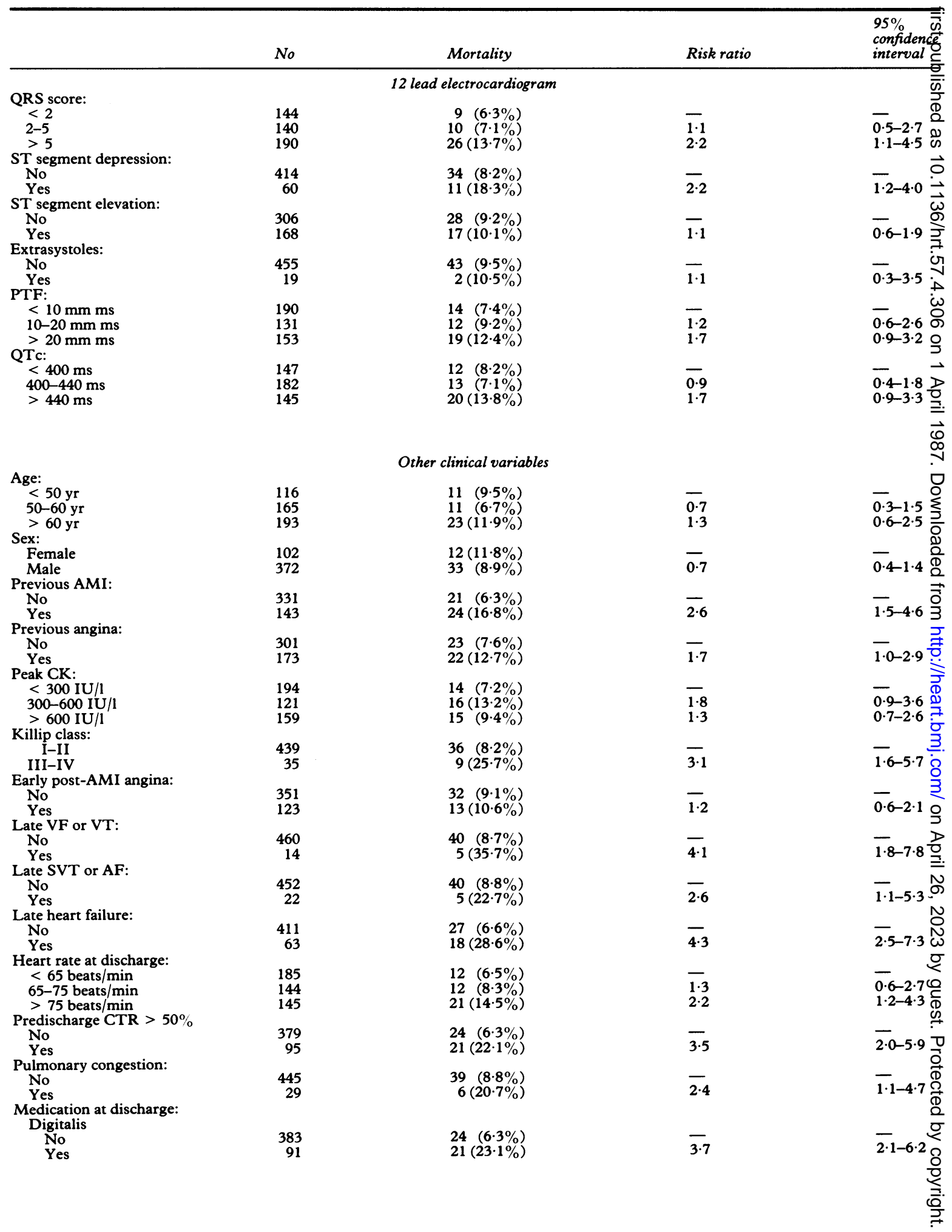




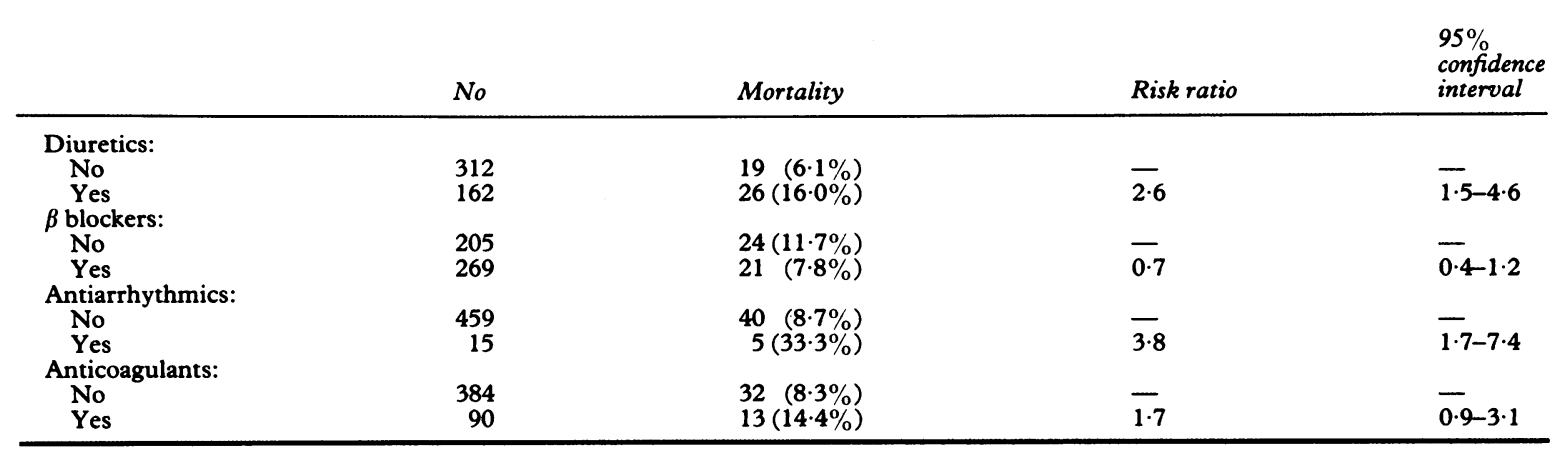

PTF, $P$ terminal force in V1; AMI, acute myocardial infarction; CK, serum creatine kinase; VF, ventricular fibrillation; VT, sustained ventricular tachycardia; SVT, paroxysmal supraventricular tachycardia; AF, paroxysmal atrial fibrillation or atrial flutter; CTR, cardiothoracic ratio.

have a value of 1 if the property considered is present and 0 if it is not. Continuous variables were divided into thirds and indicator variables for the middle and high risk categories were considered for inclusion in the model. The BMDP package was used; this selects, in a stepwise fashion, predictor variables based on the maximum likelihood ratio. This provides a measure of significance and has an asymptotic $\chi^{2}$ distribution. Thus variables were included in the model if their inclusion substantially improved the log likelihood $(p<0 \cdot 10)$ or if their removal led to a substantial decrease $(p<0 \cdot 15)$. Forward selection and backward elimination of the variables yielded the same models. Two models were developed; the first was based on the 12 lead electrocardiogram variables alone, and the other on the clinical variables alone.

The regression coefficients of each model have a direct epidemiological implication: each coefficient represents the log odds of dying when other variables in the model are controlled. Its antilogarithm is the relative risk of the event considered. As an example, if the regression coefficient for previous myocardial infarction is 1 , its antilogarithm $\left(e^{1}\right)$ is 2.7. This means that the risk of dying within one year for patients with a previous infarction is 2.7 times as high as it is for patients who had no previous myocardial infarction. The coefficients are related to the relative odds. They are a good approximation for the relative risk because mortality is rare (45 out of 474 died, $9.5 \%$ ).

\section{Results}

PREDICTION OF MORTALITY BY UNIVARIATE ANALYSIS

Table 3 gives the results of univariate analysis of the percentage mortality. Of the 12 lead electrocardiographic variables, QRS score and ST depres- sion gave statistically significant risk ratios, while QTc and P terminal force in V1 were less predictive. Of the clinical variables, previous myocardial infarction, preinfarction angina, Killip class III or IV, late paroxysmal atrial tachycardia or fibrillation, late heart failure, heart rate $>75$ beats/minute, cardiothoracic ratio $>50 \%$, and pulmonary congestion on $x$ ray were all associated with significant risk ratios. Of the drugs being taken at discharge, three reached significance-digitalis, diuretics, and antiarrhythmics.

\section{PREDICTION OF MORTALITY BY}

MULTIVARIATE ANALYSIS

Table 4 (upper part) shows the results of stepwise logistic regression of one year mortality on variables in the 12 lead electrocardiogram. QRS score $>5$, presence of ST depression, and QTc $>440 \mathrm{~ms}$ were found to be of predictive value. In particular, the presence of a QRS score $>5$ or of QTc $>440 \mathrm{~ms}$ was associated with a 1.8 fold increase in the patient's risk of dying within one year, and the presence of ST depression multiplied the risk by $2 \cdot 2$.

Table 4 (lower part) summarises the logistic regression results for the clinical variables. The presence of previous myocardial infarction, late heart failure, indication for digitalis at discharge or a cardiothoracic ratio $>50 \%$, and an indication for antiarrhythmic drugs were all independently of predictive value. As there are three variables related to pump failure: clinical signs of late heart failure, use of digitalis, and cardiothoracic ratio $>50 \%$, the construction of the model cannot be straightforward. The regression model assumes multiplicativity of the different factors. That is, if two factors are present, each with a relative risk of 3 , then the combined relative risk will be $3 \times 3=9$. As the three variables above to a large extent measure the same phenomenon, the assumption of multiplicity must 
Table 4 Variables eventually retained in the logistic function of the risk of dying within one year

\begin{tabular}{|c|c|c|c|c|c|}
\hline & $N o^{\star}$ & Coefficient & $S E$ & Relative risk & Log likelihood \\
\hline \multicolumn{6}{|l|}{12 lead ECG: } \\
\hline QRS score > 5 & 190 & $0 \cdot 6$ & $0 \cdot 3$ & $1 \cdot 8$ & \multirow{4}{*}{$-142 \cdot 2$} \\
\hline $\begin{array}{l}\text { ST segment depression } \\
\text { QTc }>440 \mathrm{~ms}\end{array}$ & $\begin{array}{r}60 \\
145\end{array}$ & $\begin{array}{l}0 \cdot 8 \\
0 \cdot 6\end{array}$ & $\begin{array}{l}0.4 \\
0.3\end{array}$ & $\begin{array}{l}2 \cdot 2 \\
1 \cdot 8\end{array}$ & \\
\hline Constant & 474 & $-2 \cdot 9$ & $0 \cdot 3$ & 1.8 & \\
\hline \multicolumn{5}{|l|}{ Clinical variables: } & \\
\hline $\begin{array}{l}\text { Previous AMI } \\
\text { Late heart failure }\end{array}$ & $\begin{array}{r}143 \\
63\end{array}$ & $1 \cdot 1$ & $\begin{array}{l}0.3 \\
0.4\end{array}$ & $\begin{array}{l}3 \cdot 0 \\
3 \cdot 0\end{array}$ & \multirow{4}{*}{$-122 \cdot 7$} \\
\hline Digitalis or CTR $>50^{\circ}$ & 148 & 1.4 & $\begin{array}{l}0.4 \\
0.4\end{array}$ & $\begin{array}{l}3 \cdot 0 \\
4 \cdot 1\end{array}$ & \\
\hline Antiarrhythmics & 15 & $1 \cdot 4$ & 0.6 & $4 \cdot 1$ & \\
\hline Constant & 474 & $-3 \cdot 7$ & $0 \cdot 3$ & - & \\
\hline
\end{tabular}

^Number of patients in whom the property considered was present.

AMI, acute myocardial infarction; CTR, cardiothoracic ratio.

be questioned. We investigated this by using interaction terms in our model. We concluded that digitalis and a cardiothoracic ratio $>50 \%$ can be combined. As a consequence, the relative risk of a patient with a cardiothoracic ratio of $>50 \%$ who also uses digitalis remains $4 \cdot 1$. If clinical signs of late heart failure are present, however, the relative risk increases to $12 \cdot 1$. This is also the case if the late heart failure is present with either a cardiothoracic ratio of $>50 \%$ or digitalis treatment.

The addition of the variables of the 12 lead electrocardiogram to the model already containing the other clinical variables gave only a marginally better fit for the data; the improvement of the log likelihood was $0.32\left(\chi^{2}=0.64, p>0.60\right)$. From this we concluded that the variables of the 12 lead electrocardiogram do not make an independent contribution to the prediction of one year mortality that is additional to that of the other clinical variables alone. Nevertheless, if clinical data are not available, the 12 lead electrocardiogram can, to a certain extent, replace them. The mean predicted probability of dying for patients who actually died is 0.12 based on the electrocardiographic model and 0.23 based on the clinical model. This demonstrates the greater ability of the clinical variables to identify patients who will die prematurely. The mean predicted probability of surviving for patients who actually survived is 0.91 based on the electrocardiographic models and 0.92 based on the clinical model, demonstrating that survival can equally well be predicted by the electrocardiographic or clinical variables.

\section{Discussion}

There is a considerable overlap between information derived from non-invasive and invasive tests when assessing the prognosis of patients after myocardial infarction. The most efficient strategy, with the highest ratio of information to cost and risk, in ano individual patient is not obvious.

We have compared the prognostic value of teststhat are not always performed at discharge, such a\& exercise testing, radionuclide ventriculography, and the 24-hour ambulatory electrocardiogram with thate of other clinical variables. ${ }^{1}$

In this study we assessed the prognostic value of the predischarge 12 lead electrocardiogram, which is routine and not expensive, to establish whether it provides information that is additional to the known clinical information and tests.

We believe that our study is the first in which a consecutive series of patients admitted to one coro-0을 nary care unit was sufficiently large to allow a multi- 3 variate analysis based on predischarge clinical and electrocardiographic variables; in which patients at relatively low risk were studied (those with conduc- $-\overrightarrow{\mathbb{D}}$ tion defects, ventricular hypertrophy and atriaff fibrillation were excluded); and which included, among other electrocardiographic variables, Selvester's QRS score ${ }^{8}$ which in the past few yearso has been increasingly used to assess the extent of myocardial damage and the prognosis. In othero studies, however, its prognostic value was not com $\rightarrow$ pared by multivariate analysis with that of other․․ electrocardiographic or clinical variables. A largen study by the Coronary Drug Project Research Group also determined the relative prognostic values of clinical and electrocardiographic variables in pa- $-\omega$ tients after infarction. ${ }^{2}$ The conclusion of that study of 2035 patients followed for three years was that STo depression $\geqslant 1 \mathrm{~mm}$ of the "ischaemic" type was theD most important independent risk predictor of all the $e^{\oplus}$ clinical and electrocardiographic findings studied, 0 including $Q$ waves, ventricular conduction defects atrial fibrillation, and extrasystoles.

We confirmed that many electrocardiographiळ variables are predictive of survival (tables 3 and 4 ) The highest risk ratios for mortality $(2 \cdot 2)$ were ob 
tained for ST segment depression. These figures accord with those reported in the Coronary Drug Project Research study. ${ }^{2}$ We, however, did not confirm the predictive value of extrasystoles and of ST elevation. This difference may be attributable to the different populations in the two studies. Patients entered the Coronary Drug Project Research study later (at least three months after the most recent infarction) and patients with ventricular conduction defects and atrial fibrillation were included. Patients were followed for longer, up to three years, in the Coronary Drug Project Research study. We studied a consecutive series of patients evaluated at hospital discharge who did not have conduction abnormalities or atrial fibrillation; follow up lasted for a year. For hospital survivors of myocardial infarction the mortality is highest in the first three to six months. ${ }^{1}$ Unlike the present study early deaths were excluded from the Coronary Drug Project Research study.

In table 2 some predischarge variables of the $\mathbf{4 7 4}$ patients in the study group are compared with those of patients excluded from the study because of ventricular conduction defects, hypertrophy, or atrial fibrillation. These data clearly indicate that patients with normal QRS pattern had much less extensive myocardial damage. This is consistent with their lower mortality during follow up. The electrocardiogram is therefore helpful in distinguishing patients without signs of conduction disturbances, ventricular hypertrophy, or atrial fibrillation-a relatively low risk group-from those in whom at least one of the above conditions is present and in whom the risk of premature death is much higher.

The QRS score and, to a lesser extent, $P$ terminal force in V1 and QTc were also predictive of mortality (table 3). QRS score is directly related to the extent of myocardial damage ${ }^{8}$ and it has been shown to correlate fairly well with left ventricular ejection fraction. In the present series the coefficient of correlation between radionuclide ejection fraction and QRS score was -0.53 (SEE $11 \%$ ) in 417 patients. The coefficient was similar $(r=-0.57$, SEE $11 \%$ ) when the analysis was limited to 296 patients with a first myocardial infarction.

Others have shown that the $\mathbf{P}$ terminal force measured from precordial lead V1 correlates with left atrial overload. ${ }^{9}$ Siltanen et al reported that the $\mathbf{P}$ terminal force was the strongest independent predictor of mortality derived from predischarge electrocardiogram in 457 post-infarction patients. ${ }^{7} \mathrm{ST}$ segment abnormalities were the second. These workers, however, did not measure the Selvester QRS score. Their population was also different and they did not exclude patients with ventricular conduction abnormalities or ventricular hypertrophy. In our study these patients had a greater $P$ terminal force in V1. This indicates more severe left ventricular dysfunction (table 2); and there was a corresponding increase in clinical signs of heart failure and a lower ejection fraction in these patients (table 2).

Mortality was higher in patients with prolonged QTc: in a seven year follow up of 55 patients with a recent infarction Schwartz and Wolf found that a prolonged QTc ( $>440 \mathrm{~ms}$ ) measured during serial electrocardiographic measurements increased the risk of sudden death by a factor of $2 \cdot 1 .{ }^{4}$ More recently, Ahnve et al confirmed that a prolonged QTc is predictive of premature death in patients after infarction. ${ }^{5}$ When they used a cut-off of $440 \mathrm{~ms}$ they found that QTc gave a sensitivity of $77 \%$ and a specificity of $84 \%$. They limited the study to patients not on medical treatment, which might influence the QTc interval.

In contrast with $Q R S$ score and $P$ terminal force in V1, which are related to pathophysiological phenomena such as the extent of myocardial damage and left atrium overload, the findings for ST depression and QTc interval are difficult to interpret. Both were predictive of mortality in our study, in which the clinically prescribed medication was not discontinued. Medication is known to be one of the many factors that affects both variables. ${ }^{5}$

In multivariate analysis ST segment depression, QTc, and QRS score were the independent predictors of mortality (table 4). Of the clinical features the most useful independent prognostic variables were persistent heart failure, a history of previous myocardial infarction, cardiomegaly on the chest $x$ ray, and the use of digitalis and antiarrhythmics at discharge. These variables all relate directly or indirectly to poor ventricular function. Several clinical and electrocardiographic variables provided useful prognostic information. When multivariate analysis was applied to the combination of clinical and electrocardiographic variables, however, the inclusion of electrocardiographic variables did not improve the prognostic information derived from the clinical data.

We conclude that the predischarge electrocardiogram can provide important prognostic information about post-infarction patients without intraventricular conduction abnormalities, ventricular hypertrophy, or atrial fibrillation, in whom clinically prescribed medication has not been discontinued, and that persistent ST segment depression, the Selvester QRS score, ${ }^{8}$ and QTc are independent predictors of survival. These electrocardiographic variables alone are not sufficiently powerful to improve risk assessment when added to other clinical information routinely collected in hospital. 


\section{References}

1 Fioretti P, Brower RW, Simoons ML, et al. The relative value of clinical variables, bicycle ergometry, resting radionuclide ventriculography and 24-hour ambulatory electrocardiographic monitoring at discharge to predict one year survival after myocardial infarction. F Am Coll Cardiol 1986;8:40-9.

2 Coronary Drug Project Research Group. The prognostic importance of the electrocardiogram after myocardial infarction. Ann Intern Med 1972;77:677-89.

3 Coronary Drug Project Research Group. Prognostic importance of premature beats following myocardial infarction. $\mathcal{F} A M A$ 1973;233:1116-24.

4 Schwartz PJ, Wolf S. QT interval prolongation as predictor of sudden death in patients with myocardial infarction. Circulation 1978;57:1074-7.

5 Ahnve S, Gilpin E, Madsen EB, et al. Prognostic importance of QT interval at discharge after acute myocardial infarction: a multicenter study of $\mathbf{8 6 5}$ patients. Am Heart $\mathcal{F}$ 1984;108:395-400.

6 Fioretti P, Brower RW, Lazzeroni E, et al. Limitations of a QRS scoring system to assess left ventricular function and prognosis at hospital discharge after myocardial infarction. Br Heart $\mathcal{f}$ 1985;53:248-52.

7 Siltanen P, Pohjola-Sintanen S, Haapakoski J, et al.
The mortality predictive power of discharge electrọ cardiogram after first acute myocardial infarction? Am Heart f 1985;109:1231-7.

8 Hindman NB, Schocken DD, Widman M, et al. Evalu $\overrightarrow{\bar{F}}$ ation of a QRS scoring system for estimating my cardial infarct size. V. Specificity and methog application of the complete system. Am $\mathcal{f}$ Cardia 1985;55:1485-90.

9 Heikkilä J, Hugenholtz PG, Tabakin BS. Prediction ơ⿱ left heart filling pressure and its sequential change if acute myocardial infarction from the terminal force. of the $\mathrm{P}$ wave. Br Heart $\mathcal{F} 1$ 1973;35:142-51.

10 Williams WL, Nair RC, Higginson LAJ, et al. Comparison of clinical and treadmill variables for the pres diction of outcome after myocardial infarction. $\mathcal{F} A$ Coll Cardiol 1984;4:477-86.

11 Simoons ML, Serruys PW, van den Brand M, et ax. Improved survival after early thrombolysis in acute myocardial infarction. Lancet 1985;ii:578-82.

12 Serruys PW, Simoons ML, Suryapranata H, et al. Pre servation of global and regional left ventricular funcs tion after early thrombolysis in acute myocardiat infarction. F Am Coll Cardiol 1986;7:729-42.

13 Simoons ML, Serruys PW, van den Brand M, et a玉 Early thrombolysis in acute myocardial infarction: limitation of infarct size and improved survival. $\mathcal{F} A$ we Coll Cardiol 1986;7:717-28. 